\title{
间斑寇蛛粗毒液的生理生化分析及两种采毒方法的比较
}

\author{
段志贵 ${ }^{1}$, 杨 静 $^{1}$, 晏晓军 ${ }^{1}$, 曾雄智 ${ }^{1}$, 王贤纯 ${ }^{1,2, *}$, 梁宋平 ${ }^{1, *}$ \\ (1. 湖南师范大学 蛋白质化学及发育生物学教育部重点实验室, 湖南 长沙 410081; \\ 2. 湖南师范大学 化学生物学及中药分析教育部重点实验室, 湖南 长沙 410081)
}

\begin{abstract}
摘要: 利用两种不同方法采集间斑寇蛛（Latrodectus tredecimguttatus）毒液并对其进行理化和生物学性质的 比较分析。结果显示, 粗毒液中的蛋白质成分主要是相对分子质量较大 $\left(>10^{4}\right)$ 的酸性蛋白质。与毒囊粗毒液相 比, 电刺激粗毒液中相对分子质量在 $10^{5}$ 左右的蛋白质含量明显高于毒囊粗毒液中的含量, 而两者中相对分子质 量较小 $\left(<10^{4}\right)$ 的蛋白质与多肽的组成非常相似。电刺激粗毒冻干粉和毒囊粗毒冻干粉对小白鼠的 $\mathrm{LD}_{50}$ 值分别为 $(0.16 \pm 0.03) \mathrm{mg} / \mathrm{kg}$ 和 $(0.39 \pm 0.05) \mathrm{mg} / \mathrm{kg}$ 体重; 对美洲蜚蠊 (Periplaneta Americana) 的 $\mathrm{LD}_{50}$ 值分别为 $1.87 \mu \mathrm{g} / \mathrm{g}$ 和 2.32 $\mu \mathrm{g} / \mathrm{g}$ 。在浓度为 $3.2 \times 10^{-6} \mathrm{~g} / \mathrm{mL}$ 时, 电刺激粗毒冻干粉能在 $(25.0 \pm 2.2) \mathrm{min}$ 内完全阻断小鼠膈神经-膈肌标本的神 经肌肉接头传递; 毒囊粗毒冻干粉在浓度为 $6 \times 10^{-6} \mathrm{~g} / \mathrm{mL}$ 时的完全阻断时间为 $(23.3 \pm 2.2) \mathrm{min}$; 粗毒液中的低相 对分子质量 $\left(<10^{4}\right)$ 部分在 $10^{-4} \mathrm{~g} / \mathrm{mL}$ 浓度下对标本的神经肌肉接头传递无明显影响。上述结果表明, 间斑寇蛛毒 液是一种富含大分子量蛋白质的混合物; 哺乳动物神经毒性主要基于其中的大的相对分子质量酸性蛋白质成分, 而不是低的相对分子质量的多肽; 电刺激粗毒液中的活性成分与毒囊粗毒液中的相似, 但含量高于毒囊粗毒液。
\end{abstract}

关键词: 间斑寇蛛; 粗毒液; 粗毒液采集; 性质; 比较分析

中图分类号: Q51；R996.3； Q959.226.2 文献标识码：A 文章编号：0254-5853-(2009)04-0381-08

\section{Venom Properties of the Spider Latrodectus tredecimguttatus and Comparison of Two Venom-Collecting Methods}

\author{
DUAN Zhi-gui ${ }^{1}$, YANG Jing ${ }^{1}$, YAN Xiao-jun ${ }^{1}$, \\ ZENG Xiong-zhi ${ }^{1}$, WANG Xian-chun ${ }^{1,2, *}$, LIANG Song-ping ${ }^{1, *}$
}

(1. Key Laboratory of Protein Chemistry and Developmental Biology of Ministry of Education of China, Hunan Normal University, Changsha 410081, China; 2. Key Laboratory of Chemical Biology and Traditional Chinese Medicine Research of Ministry of Education of China, Hunan Normal University, Changsha 410081, China)

\begin{abstract}
The bioactivities and proteinous compositions of venoms collected by two different methods from Latrodectus tredecimguttatus were analyzed and compared. Most of proteinous venom components were high-molecular-mass acidic proteins $\left(>10^{4}\right)$. Compared with the venom obtained from dissected venom gland (DGV), the venom obtained by electrical stimulation (ESV) contained more high molecular mass proteins, but the venom proteins and peptides with low molecular mass $\left(<10^{4}\right)$ in the preparations were very similar. Intraperitoneal injection of the two venoms in mice gave rise to similar poisoning symptoms and the $\mathrm{LD}_{50}$ values of ESV and DGV were $(0.16 \pm 0.03) \mathrm{mg} / \mathrm{kg}$ and $(0.39 \pm 0.05) \mathrm{mg} / \mathrm{kg}$, respectively. $\mathrm{LD}_{50}$ values of ESV and DGV in cockroaches (Periplaneta Americana) were 1.87 $\mu \mathrm{g} / \mathrm{g}$ and $2.32 \mu \mathrm{g} / \mathrm{g}$, respectively. ESV was able to block neuromuscular transmission in the mouse phrenic nerve-hemidiaphragm preparation within $(25.0 \pm 2.2) \mathrm{min}$ at a concentration of $3.2 \times 10^{-6} \mathrm{~g} / \mathrm{mL}$, whereas DGV blocked neuromuscular transmission within $(45.7 \pm 1.8) \mathrm{min}$ at the same concentration. The fraction of the venom containing proteins and peptides with molecular mass $<10^{4}$ did not obviously affect the transmission. Those results demonstrated that Latrodectus tredecimguttatus venom was a mixture rich in larger proteins rather than smaller proteins and peptides; the mammalian toxicities of the venom were due primarily to the high molecular weight acidic proteins rather than low molecular weight peptides; the active components contained in ESV and DGV were similar but the content in ESV was higher than that in DGV.
\end{abstract}


Key words: Latrodectus tredecimguttatus; Venom; Venom collection; Property; Comparative analysis

间斑寇蛛 (Latrodectus tredecimguttatus) (又名 黑葟妇蜘蛛) 毒液是由多种活性成分组成的混合 物, 是间斑寇蛛捕获猎物和防御敌害的有效武器。 在过去的几十年中，间斑寇蛛因频繁引发伤害人、 畜的事件及其毒液中大量具有药用前景的生物活 性成分的存在而引起了人们的广泛关注 (Ushkaryov et al, 2004; Isbister \& White, 2004)。研究间斑寇蛛毒 液的组成和性质对于阐明蛛毒中毒的机理、防治蜘 蛛伤害及活性毒素成分的开发利用均具有重要的 意义。将毒腺匀浆应用于龙虾神经-肌肉标本时, 发 现它能先增强然后减弱兴奋性和抑制性突触后电 位, 表明粗毒液中含有作用于龙虾神经肌肉接头突 触前膜的成分 (Kawai et al, 1972; Burmistrov et al, 1997)。将毒腺匀浆应用于青蛙神经-肌肉标本时, 发现它能够导致小终板电位频率的增加及神经末 端突触囊泡的减少(Clark et al, 1972; Fesce et al, 1986)。迄今为止, 已从间斑寇蛛毒液中分离鉴定到 包括Latrotoxins在内的 7 种主要的毒性成分。所有 研究过的Latrotoxins类毒素是一类大分子酸性蛋白 质, 相对分子质量范围分布在 $1.1 \times 10^{5} \sim 1.4 \times 10^{5}$ 之 间, 等电点分布在 $5.5 \sim 6.0$ 之间; 能导致神经末端 递质分泌的显著增加, 且对脊椎动物、昆虫或甲壳 类动物有着不同的专一性(Ushkaryov et al, 2004)。 其中被研究得最多的是 a -latrotoxin, 在有或无 $\mathrm{Ca}^{2+}$ 的条件下都可以通过诱导突触递质的大量释放而 对脊椎动物产生致命的影响(Clark et al, 1972; Fesce et al, 1986; Ceccarelli \& Hurlbut, 1980; Cavalieri et al, 1990; Orlova et al, 2000; Volynski et al, 2003; Lajus et al, 2006)。此外, Akhunov et al (1992) 和 Akhunov(1996)对间斑寇蛛 (L.tredecimguttatus) 毒 液中的激酶和两种具有血管舒缓激肽增效作用的 多肽进行了研究。他们确定了这种激酶是一种颈基 内肽酶, 这种酶能够催化切断肽内的脯氨酸羧基端 的肽键。而对大鼠的实验结果表明, 两种血管舒缓 激肽增效多肽能够延长血管舒缓激肽的抑制作用、 刺激细胞释放组氨酸和降低血压。Volkova et al (1995)从间斑寇蛛粗毒液中分离出了两种低相对分 子质量蛋白质LMWP和LMWP2, 实验证明这两种 物质对脊椎动物和蜚蠊 (Periplaneta Americana) 均 无毒性。

在蜘蛛毒素研究中, 毒液采集是重要的第一
步。然而由于间斑寇蛛的个体较小, 给采毒带来一 定困难。在间斑寇蛛毒素研究的早期, 使用最多的 毒素采集方法是分离蜘蛛毒腺后进行匀浆提取 (Volkova et al, 1995; Frontali et al, 1976; Gorio \& Mauro, 1979; Misler \& Hurlburt, 1997; Foradori et al, 2005)。尽管现在许多研究者更趋向于使用电刺激方 法来采集毒液(Graudins et al, 2001; Daly et al, 2001; Binford et al, 2003; Richardson et al, 2006), 但从离体 毒囊中抽提毒素的方法由于其操作简单而仍被经 常采用。

\section{1 材料和方法}

\section{1 材 料}

三氟乙酸（TFA）、 $\alpha$-氧基-4-羟基苯丙烯酸 $(\mathrm{HCCA}) 、$ 芥子酸 $(\mathrm{SA}) 、 乙$ 腈 $(\mathrm{ACN})$ 和二硫苏糖 醇购于 Sigma 公司。固相胶条、过硫酸铵、尿素、 琼脂糖、甘油、溴酚蓝、碘乙酰胺、硝酸银、CHAPS 和 TEMED 购于 Amersham Pharmacia Biotech 公司。 丙烯酰胺、甲叉双丙烯酰胺、Tris、甘氨酸、十二 烷基磺酸钠 (SDS) 和凝胶电泳标准蛋白质购于 Bio-Rad 公司。所有的动物实验符合国家科技部的 “实验动物的指导性意见” 和国家的相关法律。

\section{2 毒素采集}

间斑寇蛛采自中国新疆维吾尔自治区奇台县。

电刺激法: 一人将蜘蛛放在固定有铝片的塑料 平台上, 使蜘蛛腹部的尾端紧贴铝片, 将一洁净薄 塑料片置于蜘蛛的毒螯和口器之间。另一人将电刺 激器的两个电极分别放在铝片和螯肢的根部, 电极 用食盐水湿润, 以增加导电性。蜘蛛在 $25 \sim 50 \mathrm{~V}$ 、 $15 \sim 40 \mathrm{~Hz}$ 的电刺激下将无色透明的毒液分泌到薄 塑料片上。塑料片接触口器的一面用无水乙醇清洗 以避免唾液污染。收集毒液后冻干保存。此法得到 的粗毒液即为 “电刺激粗毒液”

分离毒囊法: 用镊子将督肢及毒囊拔出放入 Eppendorf管中, $\mathrm{ddH}_{2} \mathrm{O}$ 洗后去除螯肢, 用细针将毒 囊充分刺破。加入 $\mathrm{pH} 8.2 、 0.05 \mathrm{~mol} / \mathrm{L}$ 的 Tris缓冲液 (Frontali et al, 1976), 10000g离心 $10 \mathrm{~min}$ 。收集上清 并冻干保存。此法得到的粗毒液即为 “毒囊粗毒 液”。

分别称取 $1 \mathrm{mg}$ 两种粗毒冻干粉溶于 $1 \mathrm{~mL}$ $\mathrm{ddH}_{2} \mathrm{O}$ 中, $10000 \mathrm{~g}$ 离心 $10 \mathrm{~min}$, 取上清待测。采用 
Bradford法测定蛋白质含量, 以BSA为标准蛋白质 制定标准曲线, 测定粗毒液溶液的浓度, 得出电刺 激粗毒冻干粉和毒囊粗毒冻干粉的蛋白质含量分 别为 $55.2 \%$ 和 $37.0 \%$ 。

\section{3 半致死剂量的测定}

小鼠半致死剂量的测定参照 Schweitz (1984)及 Liang et al (1993)的方法进行。准确称重的毒素溶解 于生理盐水后 $10000 \mathrm{~g}$ 离心 $10 \mathrm{~min}$, 收集上清。测 定毒囊粗毒液半致死剂量时, 36 只昆明种小鼠随机 分成 6 组, 其中五组分别按照 $0.608 、 0.468 、 0.360$ 、 0.277 和 $0.213 \mathrm{mg} / \mathrm{kg}$ 的剂量每只腹腔注射 $20 \mu \mathrm{L}$ 毒 液, 并设生理盐水对照组。测定电刺激粗毒液半致 死剂量时, 54 只昆明种小鼠随机分成 9 组, 其中 8 组分别按照 $0.359 、 0.276 、 0.213,0.163,0.126$ 、 $0.097 、 0.074$ 和 $0.057 \mathrm{mg} / \mathrm{kg}$ 的剂量每只腹腔注射 20 $\mu \mathrm{L}$ 毒液, 设生理盐水对照组。观察每组小鼠 $24 \mathrm{~h}$ 内的死亡率, 根据每组的死亡率计算半致死剂量。

粗毒液对蜚蠊 $\mathrm{LD}_{50}$ 的测定参照Schweitz (1984) 的方法进行。用美洲蜚蠊按每克体重 $0.951 、 1.521$ 、 $2.434 、 3.894$ 和 $6.230 \mu \mathrm{g}$ 的剂量从第四、五腹板交接 处注入腹腔, 对照组为等体积的生理盐水, 观察 24 $\mathrm{h}$ 内的死亡率。

\section{4 膈神经膈肌实验}

实验在离体的小鼠膈神经-膈肌标本上进行 (Zhou et al, 1997)。昆明种小鼠颈部脱位致死后, 迅 速分离膈神经膈肌并放入盛有台氏液的标本槽中, $30 \sim 32^{\circ} \mathrm{C}$ 恒温并持续供氧 $\left(95 \% \mathrm{O}_{2} / 5 \% \mathrm{CO}_{2}\right)$ 。用铂丝 吸附电极刺激膈神经 (超强刺激, 频率 $0.2 \mathrm{~Hz}$, 持 续 $2 \mathrm{~ms}$, 方波), 机-电转换器将张力信号转换成电 信号后被信号处理系统 (Model Biolap 98, 中国) 记 录下来。

\section{5 粗毒液的 RP-HPLC 分析}

RP-HPLC在Waters 公司的Alliance HPLC仪器 上进行, 所用分析柱为反相 $\mathrm{C} 4$ 柱 $(4.6 \mathrm{~mm} \times 250 \mathrm{~mm}$, 依利特公司), 检测器为 996 光检测器。流动相 $\mathrm{A}$ 为 含 $0.1 \% \mathrm{TFA}$ 的 $\mathrm{ddH}_{2} \mathrm{O}$; 流动相B为含 $0.1 \% \mathrm{TFA}$ 的 $\mathrm{ACN}$ 。洗脱梯度为 $0 \sim 45 \mathrm{~min}, 0 \sim 90 \% \mathrm{~B}$; 流速 0.7 $\mathrm{mL} / \mathrm{min} ; 280 \mathrm{~nm}$ 检测。每次进样量均为 $1 \mathrm{mg}$ 粗毒冻 干粉。

\section{6 质谱分析}

质谱分析在 Ultraflex TOF/TOF (Bruker Daltonics) 上进行, 线性操作模式。线性检测器电 压、离子源 1 、离子源 2 和离子源棱镜电压分别为
$1.642 \mathrm{~V} 、 25 \mathrm{~V} 、 23.4 \mathrm{~V}$ 和 $6.0 \mathrm{~V}$, 激光频率 $50 \mathrm{~Hz}$, 外标校正。

\subsection{SDS-PAGE 分析}

两种粗毒液的 SDS-PAGE 分析参照 Laemmli (1970)的方法在 $12 \%$ 的变性聚丙烯酰胺凝胶上进 行。两种冻干的粗毒冻干粉各 $70 \mu \mathrm{g}$, 分别溶解在 $70 \mu \mathrm{L}$ 样品溶解液 $(50 \mathrm{mmol} / \mathrm{L}$ Tris- $\mathrm{HCl}, \mathrm{pH} 6.8,65$ $\mathrm{mmol} / \mathrm{L}$ DTT, $0.5 \mathrm{mmol} / \mathrm{L}$ PMSF, $2 \% \mathrm{SDS}$ 及痕量的 溴酚蓝）中, 沸水浴 $3 \mathrm{~min}$ 。冷却后在 $10000 \mathrm{~g}$ 转速 下离心 $15 \mathrm{~min}$, 取上清液加入上样槽, 以低相对分 子质量标准蛋白质作为对照。浓缩胶的电泳电流为 $20 \mathrm{~mA}$, 分离胶的电泳电流为 $40 \mathrm{~mA}$ 。电泳完成后银 染显色。

\section{8 双向凝胶电泳}

双向凝胶电泳参照 Bjellqvist (Bjellqvist et al, 1993)的方法进行。 $250 \mu \mathrm{g}$ 粗毒冻干粉溶解在 $350 \mu \mathrm{L}$ 水化液中 $[8 \mathrm{~mol} / \mathrm{L}$ urea, $4 \%$ CHAPS (w/v), $65 \mathrm{mmol} / \mathrm{L}$ DTT, $0.5 \%$ IPG buffer (v/v), $0.5 \%$ pharmalyte, 痕量 溴酚蓝], $10000 \mathrm{~g}$ 离心 $10 \mathrm{~min}$ 。上清液加至放有 18

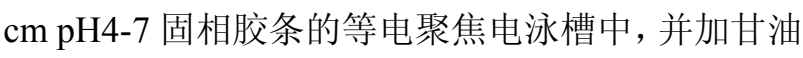
覆盖。按照 $30 \mathrm{~V} 14 \mathrm{~h}, 500 \mathrm{~V} 1 \mathrm{~h}, 1000 \mathrm{~V} 1 \mathrm{~h}, 8000 \mathrm{~V}$ 至 $22500 \mathrm{Vh}$ 的梯度等电聚焦电泳过夜。后将载有毒 素蛋白质的胶条先后在平衡液 A $(0.05 \mathrm{~mol} / \mathrm{L}$ Tris-HCl, pH6.8, 8mol/L urea, 30\% glycerol, $1 \%$ SDS 和 $0.2 \%$ DTT $)$ 和平衡液 B $(0.05 \mathrm{~mol} / \mathrm{L}$ Tris- $\mathrm{HCl}$, pH6.8, $8 \mathrm{~mol} / \mathrm{L}$ urea, $30 \%$ glycerol, $1 \%$ SDS, 3\% 碘 乙酰胺, 痕量澳酚蓝)中平衡 $15 \mathrm{~min}$ 。随后将胶条转 至第二向凝胶。在 5\%浓缩胶中电泳的电流为 20 $\mathrm{mA}, 12 \%$ 分离胶中电泳的电流为 $50 \mathrm{~mA}$, 电泳温度 $10^{\circ} \mathrm{C}$ 。待澳酚蓝线电泳至距离凝胶末端 $1 \mathrm{~cm}$ 处时停 止, 分离后的蛋白质银染显色。

\section{2 结 果}

\section{1 粗毒液的毒性}

小鼠腹腔注射两种方法采集的粗毒液后, 中毒 症状非常相似, 主要有四肢㿈软、多汗、惊厥、不 进食、呼吸急促、睁眼困难和小便失禁等。而生理 盐水对照组小鼠的行为正常。电刺激粗毒冻干粉和 毒囊粗毒冻干粉的 $\mathrm{LD}_{50}$ 分别为 $(0.16 \pm 0.03) \mathrm{mg} / \mathrm{kg}$ 和 $(0.39 \pm 0.05) \mathrm{mg} / \mathrm{kg}$, 而对美洲蜚蠊的 $\mathrm{LD}_{50}$ 值则 分别为 $1.87 \mu \mathrm{g} / \mathrm{g}$ 和 $2.32 \mu \mathrm{g} / \mathrm{g}$ 。这些结果显示, 粗毒 液中含有针对哺乳动物和昆虫的活性毒素成分且 电刺激粗毒液的毒性成分含量更高。 


\section{2 粗毒液对膈神经-膈肌标中神经肌肉接头传递 的影响}

浸在台氏液中的膈神经-膈肌标本在刺激过程 中, 其张力信号无明显改变(图 1A)。加入粗毒液后, 刺激膈神经引起的张力信号逐渐减弱, 直至完全消 失, 提示神经肌肉接头传递被完全阻断。完全阻断 后, 对直接刺激膈肌引起的收缩并无明显影响(图 $1 \mathrm{E})$ 。两种粗毒液都能阻断标本的神经肌肉接头传 递, 但两种粗毒液的活性水平存在差异。毒囊粗毒 冻干粉浓度为 $3.2 \mu \mathrm{g} / \mathrm{mL}$ 时, 完全阻断神经肌肉接 头传递的时间为 $(45.7 \pm 1.8) \mathrm{min}$ (图 1B), 而相同 浓度的电刺激粗毒冻干粉在 $(25.0 \pm 2.2) \mathrm{min}$ 内即 可完全阻断之(图 1C), 说明电刺激粗毒冻干粉的活
性更高 $(P<0.01)$ 。此外,相同实验条件下粗毒冻干 粉的低相对分子质量成分 $\left(<10^{4}\right)$ 在浓度高达 100 $\mu \mathrm{g} / \mathrm{mL}$ (合并反相峰冻干后称量配制) 时也未能对标 本的神经肌肉接头的传递产生明显的影响(图 1D)。

\subsection{RP-HPLC 分析}

RP-HPLC 分析显示,两种粗毒液主要成分的出 峰时间大约分布在 5 和 $13 \mathrm{~min}$ 左右和 $20 \sim 40 \mathrm{~min}$ 之间 (图 2)。但可明显看出, 毒囊粗毒液成分 (图 2B) 要比电刺激粗毒液 (图 2A) 中的复杂, 在 5 $15 \mathrm{~min}$ 范围内从反相柱上洗脱的成分比较多, 提示 毒囊粗毒液中含有更多的亲水性较强的小分子物 质; 在 $30 \mathrm{~min}$ 以后出现更多的洗脱峰, 说明毒囊粗 毒液含有的疏水性较强的大分子成分多于电刺激 粗毒液。

\section{A}

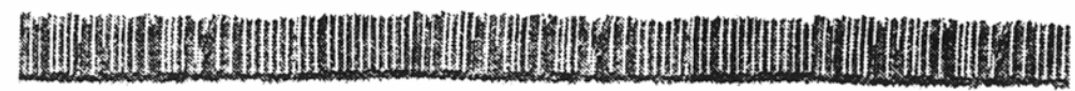

Adding gland puncture venom

E

$\mathrm{B}$

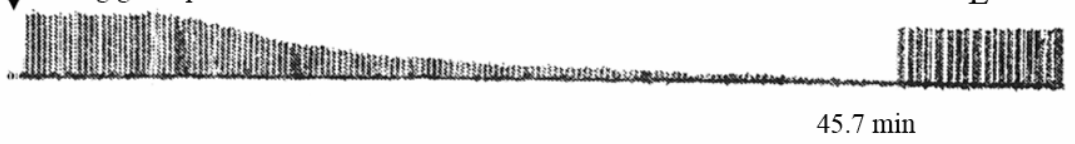

C

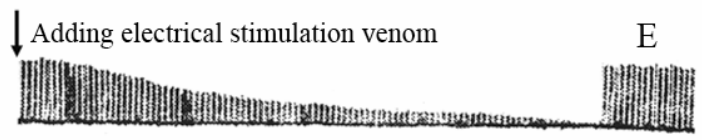

$25.0 \mathrm{~min}$

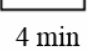

$\downarrow$ Adding low-molecular-mass fraction

$\mathrm{D}$

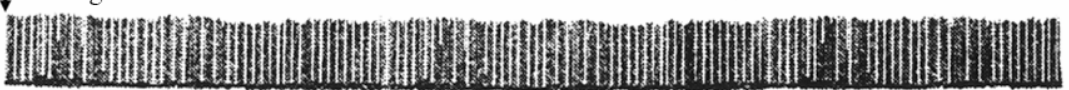

图 1 粗毒液对小鼠离体膈神经-膈肌的神经肌肉接头传递的影响

Fig. 1 Effects of venoms on neuromuscular transmission in isolated mouse phrenic nerve-hemidiaphragm A：对照; B: 毒囊粗毒冻干粉浓度为 $3.2 \times 10^{-6} \mathrm{~g} / \mathrm{mL} ; \mathrm{C}$ : 电刺激粗毒冻干粉浓度为 $3.2 \times 10^{-6} \mathrm{~g} / \mathrm{mL} ; \mathrm{D}$ : 低相对分子质量成分浓度为 $1 \times$ $10^{-4} \mathrm{~g} / \mathrm{mL} ; \mathrm{E}$ : 完全阻断后直接刺激膈肌。

A: Control; B: $3.2 \times 10^{-6} \mathrm{~g} / \mathrm{mL}$ of venom obtained from dissected venom gland; C: $3.2 \times 10^{-6} \mathrm{~g} / \mathrm{mL}$ of venom obtained by electrical stimulation; D: $10^{-4} \mathrm{~g} / \mathrm{mL}$ of low-molecular-mass fraction; E: Direct stimulation on hemidiaphragm after blockade.
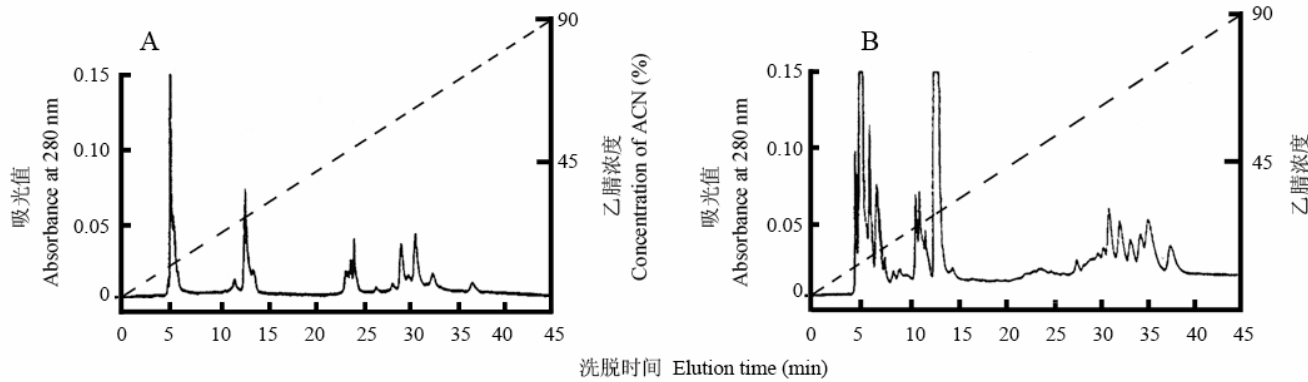

图 2 两种方法采集间斑寇蛛粗毒液的 RP-HPLC 图

Fig. 2 RP-HPLC chromatograms of venoms collected from Latrodectus tredecimguttatus by two different methods A：电刺激粗毒液; B: 毒囊粗毒液。A: Venom obtained by electrical stimulation; B: Venom obtained from dissected venom gland. 


\section{4 相对分子质量低于 $10^{4}$ 的多肽组分的质谱分析} 质谱分析结果显示，两种粗毒液的多肽成分相 似，且相对分子质量低于 $10^{4}$ 且丰度较高的多肽组分
都不多 (图 3)。主要多肽成分的相对分子质量分布 在 $6 \times 10^{6} \sim 8 \times 10^{6}$ 的范围内, 其中两种主要成分的相 对分子质量 $\left(\mathrm{M}+\mathrm{H}^{+}\right)$分别为 7823 和 7950 。
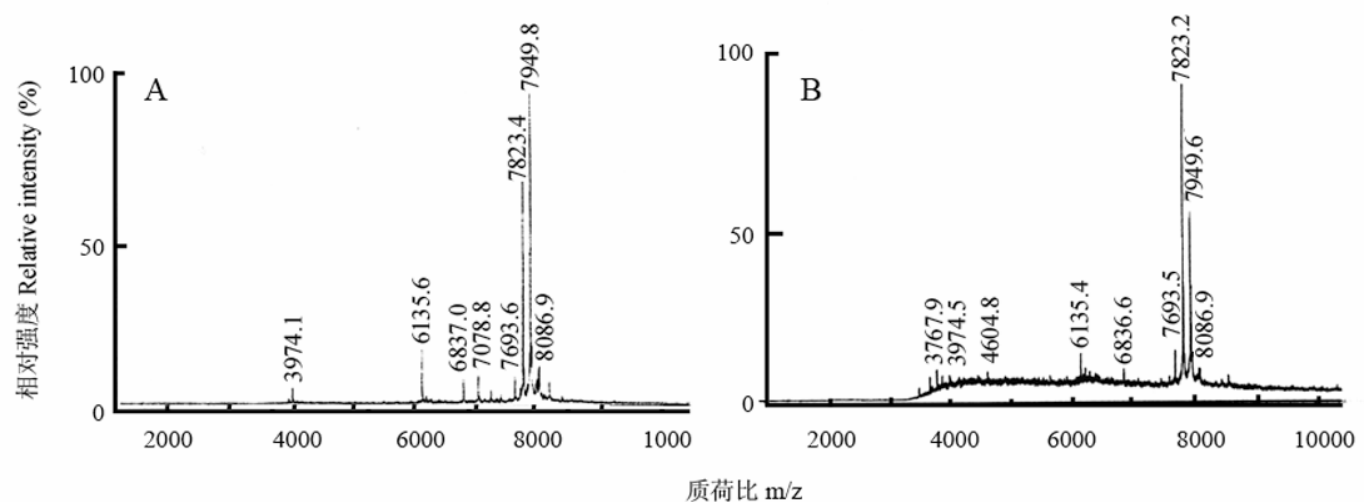

图 3 两种方法采集的间斑寇蛛粗毒液中低相对分子质量部分的质谱分析图

Fig. 3 Mass spectrometric analysis of low- molecular fraction of venoms collected from Latrodectus tredecimguttatus by two different methods

A: 电刺激粗毒液; B: 毒囊粗毒液。

A: Venom obtained by electrical stimulation; B: Venom obtained from dissected venom gland.

\subsection{SDS-PAGE 分析}

粗毒液的 SDS-PAGE 图谱如图 4 所示。两种粗

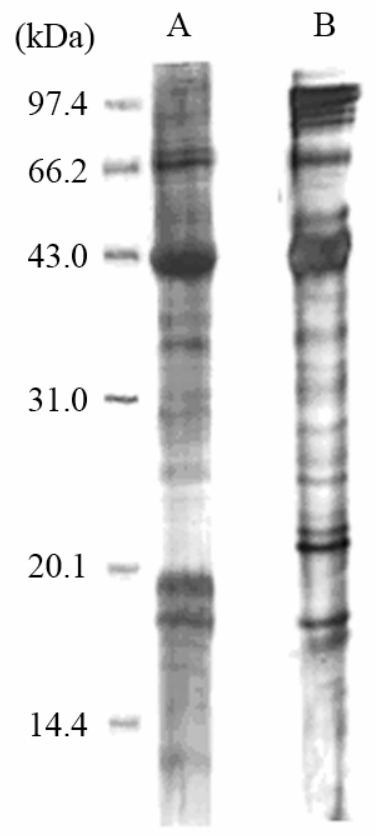

图 4 两种方法采集的间斑寇蛛粗毒液 SDS-PAGE 图

Fig. 4 SDS-PAGE gel images of venoms collected from Latrodectus tredecimguttatus by two different methods

A：电刺激粗毒液; B: 毒囊粗毒液。

A: Venom obtained by electrical stimulation; B: Venom obtained from dissected venom gland.
毒液中的蛋白质的相对分子质量大多在 $10^{4}$ 以上。 毒囊粗毒液的凝胶电泳图像不如电刺激粗毒液的 清晰，提示前者的组成更为复杂，可能存在脂质污 染等。相对于毒囊粗毒液来说，电刺激粗毒液有更 多的蛋白质分布，其相对分子质量在 $10^{5}$ 左右。

\section{6 双向凝胶电泳分析}

在采用宽等电点范围（pI 3 10）胶条的双向 凝胶电泳的预实验中发现, 大多数的粗毒液蛋白质 分布在酸性范围中（图谱未列出）。因此，我们选 择 $\mathrm{pH} 4 \sim 7$ 的 IPG 胶条以更好地分离粗毒液蛋白质

(图 5)。图谱分析软件显示, 电刺激粗毒液的蛋白 质点少于 250 个, 而毒囊粗毒液的蛋白质点超过 500

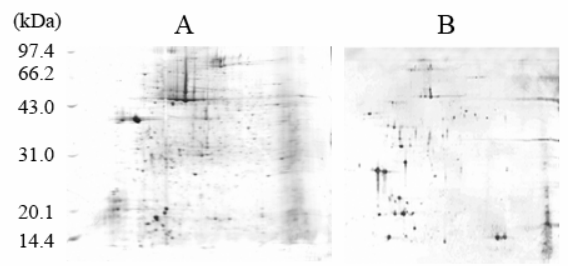

图 5 两种方法采集的间斑寇蛛粗毒液的双向凝胶电泳图

Fig. 5 2-DE gel images of venoms collected from Latrodectus tredecimguttatus by two different methods

A：电刺激粗毒液; B: 毒囊粗毒液。

A: Venom obtained by electrical stimulation; B: Venom obtained from dissected venom gland. 
个, 说明从分离的毒囊抽提毒素时被更多的非毒素 蛋白质所污染。此外, 从凝胶图像可以看出, 大多 数的蛋白质的相对分子质量分布在 $10^{4} \sim 6.6 \times 10^{4}$ 的 范围中, 其相对分子质量在大于 $6.6 \times 10^{4}$ 的区域里 蛋白质电较少, 这表明一些大分子量蛋白质在等点 聚焦电泳过程中被丢失，未能进入二向凝胶。

\section{3 讨 论}

全世界每年都发生许多被 Lactrodectus 属间斑 寇蛛蜘蛛咬伤的中毒事件。尽管导致人员死亡的事 件很少发生, 但是如果没有得到及时的治疗, 使病 人饱受痛苦的中毒症状可能要持续几天乃至几个 星期。由于难以得到足够量的间斑蜘蛛毒素, 妨碍 了毒素研究的系统和深入。所以至今为止, 对其毒 素成分和中毒机理尚缺乏全面的了解。早期研究通 常采用的采毒方法是匀浆离体毒囊后抽提毒素 (Volkova et al, 1995; Frontali et al, 1976; Gorio \& Mauro, 1979; Misler \& Hurlburt, 1997; Foradori et al, 2005)。显然, 采用此法得到的粗毒液不可避免的被 大量的非毒素蛋白质所污染。本研究中, 毒囊粗毒 液的蛋白质含量 $(37.0 \%)$ 比电刺激粗毒液要低 $(55.2 \%)$, 说明采用穿刺分离毒囊后再提取的方法 获取毒素时可能被除组织蛋白质外的非蛋白质成 分的污染, 同时缓冲液的离子成分的存在也降低了 粗毒液的蛋白质含量。但采用穿刺分离毒囊后再提 取的方法获取毒素可以降低污染程度, 文献报道的 毒囊匀浆法提取的间斑寇蛛粗毒液的半致死剂量

(0.59 mg/kg) (McCrone, 1964)高于本文毒囊穿刺 法得到的粗毒液半致死剂量 $(0.39 \mathrm{mg} / \mathrm{kg})$ 证明了这 一点。

由于分离毒囊获取毒素的方法无法避免污染 且不能重复利用宝贵的蜘蛛毒素资源。所以, 许多 研究尝试从活体动物采集毒液的各种方法, 其中用 得较多的是电刺激法 (Liu et al, 1995; Graudins et al, 2001; Daly et al, 2001; Binford et al, 2003; Richardson et al, 2006)。然而, 电刺激采毒法在蜘蛛 毒液的采集中也遇到很多的挑战, 如难以从个体较 小的蜘蛛采集毒液、有可能遭受唾液污染等。由于 蜘蛛不能进食固体食物而采用体外消化, 将具有消 化能力的唾液注入到猎物中消化猎物, 然后吸入液 化了的营养物质。自然状态下尽管口器与鳌肢非常 接近, 但是消化液与毒液还是很少混合, 这是因为 蜘蛛在注入消化液之前已主要靠毒液和蜘蛛丝控
制猎物。然而, 电刺激采集毒液时则容易同时刺激 消化液的分泌, 如不采取适当措施, 消化液中的许 多蛋白酶和其它酶可能降解毒素成分。在本研究 中, 建立了一种适合小型蜘蛛活体采毒的电刺激方 法, 且在给予电刺激之前将一洁净薄塑料片置于口 器与螯肢之间, 既可以有效防止消化液的污染, 又 方便微量注射器或移液器收集毒液。用这种方法采 集的毒液未受组织蛋白和其他成分的污染, 所以更 能代表纯的蜘蛛毒液, 更适合用来研究间斑寇蛛粗 毒液的理化和生物学性质。

液相色谱、凝胶电泳和生物质谱等分析技术相 结合可以帮助我们较为全面地了解问斑寇蛛毒液 的组成, 尤其是其中的蛋白质和多肽成分。分析结 果表明, 间斑寇蛛粗毒液主要由相对分子质量较大 的酸性蛋白质组成, 相对分子质量 $10^{4}$ 以下的蛋白 质和多肽较少。SDS-PAGE分析结果显示, 电刺激 粗毒液中相对分子质量在 $10^{5}$ 左右的蛋白质含量高 于毒囊粗毒液, 提示相对分子量在 $10^{5}$ 以上的代表 性Latrotoxins类毒素蛋白(Grishin, 1998)在电刺激粗 毒液中的含量高于毒囊粗毒液中的含量。双向凝胶 电泳分离粗毒液时, 两种粗毒液的相对分子量在 $10^{5}$ 的附近区域均只有很少的蛋白质点出现。这表明 部分大相对分子质量蛋白质在等电聚焦电泳过程 中被丢失, 提示SDS-PAGE比双向凝胶电泳更适合 分析间斑寇蛛的粗毒液。结合质谱分析结果可知, 两种粗毒液的不同主要反映在大相对分子质量蛋 白质成分的含量上, 而相对分子质量低于 $10^{4}$ 且丰 度较高的蛋白质和多肽成分的组成则非常相似。

分别给小鼠和蜚蠊注射两种粗毒液时, 均能引 起多种中毒症状, 且剂量较高时引起动物死亡, 提 示粗毒液中含有针对哺乳动物和昆虫的毒性成分。

$\mathrm{LD}_{50}$ 值的差异提示, 两种方法采集的粗毒液含有相 似的作用于哺乳动物和昆虫的活性成分, 只是含量 存在差异。离体膈神经-膈肌标本的神经肌肉接头阻 断实验也证明两种粗毒液具有相似的生物学活性, 只是活性成分含量的不同导致了有效剂量的差异。 由于粗毒液的低相对分子质量部分不能阻断离体 膈神经-膈肌的神经肌肉接头传递, 因此可以推断, 间斑寇蛛粗毒液的哺乳动物毒性主要依赖于其高 相对分子质量的蛋白质成分而不是低相对分子质 量的蛋白质和多肽, 这与Selenocosmia huwena (Liang, 2004)和Macrotheleraveni raveni (Zeng et al, 2003)的粗毒液不同, 这些蜘蛛粗毒液毒性的发挥与 
其含有的大量生物活性多肽密切相关。此外, 尽管 毒囊粗毒冻干粉因有部分非毒素蛋白质和其他成 分的污染, 其小鼠半致死剂量 $(0.39 \mathrm{mg} / \mathrm{kg})$ 高于电 刺激粗毒冻干粉 $(0.16 \mathrm{mg} / \mathrm{kg})$, 但仍低于许多其他 种类蜘蛛粗毒冻干粉的小鼠半致死剂量, 如 $S$. huwena $(1.16 \mathrm{mg} / \mathrm{kg}$ ) (Liang et al, 1993), M. raveni $(2.85 \mathrm{mg} / \mathrm{kg})$ (Zeng et al, 2003), L. gaucho (0.74 $\mathrm{mg} / \mathrm{kg})$, L. laeta $(1.45 \mathrm{mg} / \mathrm{kg})$ 和 L. intermedia $(0.48$ $\mathrm{mg} / \mathrm{kg}$ ) (Barbaro et al, 1996), 表明间斑寇蛛的确是 世界上毒性最强的蜘蛛之一。

本研究首次对两种方法采集的间斑寇蛛粗毒

\section{参考文献:}

Akhunov AA, Golubenko Z, Sosnina N. 1992. Isolation and characterization of biological properties of inhibitors angiotensin-1-converting enzyme from the spider venom Latrodectus tredecimguttatus[J]. Agents Actions Suppl, 38(1): 469-474.

Akhunov AA, Makevnina LG, Golubenko Z, paskhina, TS. 1996. Kininase of the Latrodectus tredecimguttatus venom: a study of its enzyme substrate specificity[J]. Immunopharmacology, 32(1-3): 160-162.

Barbaro KC, Ferreira ML, Cardoso DF, Eickstedt VR, Mota I. 1996. Identification and neutralization of biological activities in the venoms of Loxosceles spiders[J]. Braz J Med Biol Res, 29(11): 1491-1497.

Binford GJ, Wells MA. 2003. The phylogenetic distribution of sphingomyelinase D activity in venoms of Haplogyne spiders[J]. Comp Biochem Physiol B, 135(1): 25-33.

Bjellqvist B, Sanchez JC, Pasquali C, Ravier F, Paquet N, Frutiger S, Hughes GJ, Hochstrasser D. 1993. Micropreparative two-dimensional electrophoresis allowing the separation of samples containing milligram amounts of protein[J]. Electrophoresis, 14(2): 1375-1378.

Burmistrov YM, Shuranova ZP, Artiukhina NI. 1997. Effects of black widow spider venom and latrocrustatoxin on crustacean nerve cells: electrophysiological and ultrastructural study[J]. Gen Pharmacol, 28(1): 159-166.

Cavalieri M, Corvaja N, Grasso A. 1990. Immunocytological localization by monoclonal antibodies of alpha-latrotoxin in the venom gland of the spider Latrodectus tredecimguttatus[J]. Toxicon, 28(3): 341-346.

Ceccarelli B, Hurlbut WP. 1980. $\mathrm{Ca}^{2+}$-dependent recycling of synaptic vesicles at the frog neuromuscular junction[J]. J Cell Biol, 87(1): 297-303.

Clark AW, Hurlbut WP, Mauro A. 1972. Changes in the fine structure of the neuromuscular junction of the frog caused by black widow spider venom[J]. J Cell Biol, 52(1): 1-14.

Daly FFS, Hill RE, Bogdan GM, Dart RC. 2001. Neutralization of Latrodectus mactans and $L$. hesperus venom by redback spider ( $L$. hasseltii) antivenom[J]. Clin Toxicol, 39(2): 119-123.

Fesce R, Segal JR, Ceccarelli B, Hurlbut WP. 1986. Effects of black widow spider venom and $\mathrm{Ca}^{2+}$ on quantal secretion at the frog neuromuscular junction[J]. J Gen Physiol, 88(1): 59-81.

Foradori MJ, Smith SC, Smith E, Wells RE. 2005. Survey for potentially necrotiziing spider venoms, with special emphasis on Cheiracathium mildei[J]. Comp Biochem Physiol C, 141(1): 32-39.

Frontali N, Ceccarelli B, Gorio A, Mauro A, Siekevitz P, Tzeng MC, Hurlbut WP. 1976. Purification from black widow spider venom of a
液进行了生理生化性质的比较分析, 阐明了间斑寇 蛛粗毒液的一些重要的理化和生物学性质, 并对两 种采毒方法进行了系统的评估。通过比较发现, 两 种方法均能提取到间斑寇蛛毒液; 但本研究采用的 针对小型蜘蛛的电刺激采毒法能得到纯的蜘蛛毒 液, 是目前最理想的采集粗毒液的方法之一。由于 穿刺分离毒囊后再提取的方法在原有的毒囊匀浆 法上进行了改进，降低了污染程度的同时具有简 便、快捷的优点。且间斑寇蛛成蛛一般在入冬前会 自然死去，在其死去之前分离其毒囊可获得一定量 的毒素。因此, 这种采毒方法仍有一定的应用价值。

protein factor causing the depletion of synaptic vesicles at neuromuscular junctions[J]. J Cell Biol, 68(3): 462-479.

Gorio A, Mauro A. 1979. Reversibility and mode of action of black widow spider venom on the vertebrate neuromuscular junction[J]. $J$ Gen Physiol, 73(2): 245-263.

Graudins A, Padula M, Broady K, Nicholson GM. 2001. Red-back spider (Latrodectus hasselti) antivenom prevents the toxicity of widow spider venoms[J]. Ann Emerg Med, 37(2): 154-160.

Grishin EV. 1998. Black widow spider toxins: the present and the future[J]. Toxicon, 36(11): 1693-1701.

Isbister GK, White J. 2004. Clinical consequences of spider bites: recent advances in our understanding[J]. Toxicon, 43(5): 477-492.

Kawai N, Mauro A, Grundfest H. 1972. Effect of black widow spider venom on the lobster neuromuscular junction[J]. J Gen Physiol, 60(6): 650-664.

Laemmli UK. 1970. Cleavage of structural proteins during the assembly of the head of bacteriophage T4[J]. Nature, 227(5259): 680-685.

Lajus S, Vacher P, Huber D, Dubois M, Benassy MN, Ushkaryov Y, Lang J. 2006. a -latrotoxin induces exocytosis by inhibition of voltage-dependent $\mathrm{K}+$ channels and by stimulation of L-type $\mathrm{Ca}^{2+}$ channels via latrophilin in $\beta$-cells[J]. J Biol Chem, 281(9): 5522-5531.

Liang SP, Qin YB, Zhang DY, Pan X, Chen XD, Xie JY. 1993. Biological characterization of spider (Selenocosmia huwena) crude venom[J]. Zool Res, 14(1): 60-65.

Liang SP. 2004. An overview of peptide toxins from venom of the Chinese bird spider Selenocosmia huwena Wang [=Ornithoctonus huwena(Wang)][J]. Toxicon, 43: 575-585.

Liu YS, Wang X, Jin L, Li HM, Wang DC. 1995. The purification and comparison of Chinese scorpion neurotoxins from different locations[J]. Zool Res, 16(3): 263-270. [刘延顺，王 金金，金 雷，李宏民，王大 成. 1995. 不同产地中华马氏钳蝎神经毒素的分离纯化和部分性质 比较研究. 动物学研究, 16(3): 263-270.]

McCrone JD. 1964. Comparative lethality of several Latrodectus venoms[J]. Toxicon, 2: 201-203.

Misler S, Hurlbut WP. 1997. Action of black widow spider venom on quantized release of acetylcholine at the frog neuromuscular junction: Dependence upon external $\mathrm{Mg}^{2+}[\mathrm{J}]$. Proc Natl Acad Sci USA, 76(2): 991-995.

Orlova EV, Rahman MA, Gowen B, Volvnski KE, Ashton AC, Manser C, Van Heel M, Ushkaryov YA. 2000. Structure of $\alpha$-latrotoxin oligomers 
reveals that divalent cation-dependent tetramers form membrane pores[J]. Nat Struct Biol, 7(1): 48-53.

Richardson M, Pimenta AMC, Bemquerer MP, Santoro MM, Beirao PS, Lima ME, Fiqueiredo SG, Bloch C Jr, Vasconcelos EA, Campos FA, Gomes PC, Cordeiro MN. 2006. Comparison of the partial proteomes of the venoms of Brazilian spiders of the genus Phoneutria[J]. Comp Biochem Physiol C, 142(3-4): 173-187.

Schweitz H. 1984. Lethal potency in mice of toxins from scorpion, sea anemone, snake and bee venoms following intraperitoneal and intracisternal injection[J]. Toxicon, 22(2): 308-311.

Ushkaryov YA, Volynski KE, Ashton AC. 2004. The multiple actions of black widow spider toxins and their selective use in neurosecretion studies[J]. Toxicon, 43(5): 527-542.

Volynski KE, Capogna M, Ashton AC, Thomson D, Orlova EV, Manser CF,
Ribchester RR, Ushkaryov YA. 2003. Mutant $\alpha$-Latrotoxin $\left(\mathrm{LTX}^{\mathrm{N} 4 \mathrm{C}}\right)$ does not form pores and causes secretion by receptor stimulation[J]. $J$ Biol Chem, 278(33): 31058-31066.

Volkova TM, Pluzhnikov KA, Woll PG, Grishin EV. 1995. Low molecular weight components from black widow spider venom[J]. Toxicon, 33(4) 483-489.

Zeng XZ, Xiao QB, Liang SP. 2003. Purification and characterization of raventoxin-I and raventoxin-III, two neurotoxic peptides from the venom of the spider Macrotheleraveni[J]. Toxicon, 41(6): 651-656.

Zhou PA, Xie XJ, Li M, Yang DM, Xie ZP, Zong X, Liang SP. 1997. Blockade of neuromuscular transmission by huwentoxin-I, purified from the venom of the Chinese bird spider Selenocosmia huwena[J]. Toxicon, 35(1): 39-45. 